\title{
ELEVAÇÃO DOS NÍVEIS SÉRICOS DE LAMININA NA CIRROSE HEPÁTICA INDUZIDA PELO TETRACLORETO DE CARBONO
}

\author{
Lindalva Batista NEVES, Regina Maria CATARINO, Maria Regina Regis SILVA e Edison Roberto PARISE
}

\begin{abstract}
RESUMO - Racional - Laminina sérica tem sido correlacionada com a hipertensão portal e a capilarização dos sinusóides na doença hepática crônica. Pouco é conhecido sobre sua dinâmica nas doenças hepáticas. Objetivos - Estudar os níveis séricos de laminina e correlacioná-los com graus de fibrose hepática e hipertensão portal em ratos tratados com tetracloreto de carbono. Material e Métodos - Quarenta e nove ratos albinos Wistar foram mantidos como controles $(\mathrm{n}=16)$ ou tratados com solução de tetracloreto de carbono a $8 \%$ $(\mathrm{n}=23)$. Entre a $6^{\mathrm{a}} \mathrm{e} 16^{\mathrm{a}}$ semana de experimento animais foram sacrificados após cateterização da veia porta e medida da pressão portal Fragmentos de fígado foram fixados em formol para microscopia óptica. A fibrose hepática foi classificada em perivenular, septal incompleta e completa e cirrose. Concentração de laminina circulante foi determinada pela técnica de ensaio imunoenzimático com anticorpo desenvolvido a partir de laminina isolada de tumor Engelbreth-Holm-Swarm e purificado em coluna de afinidade. Resultados - A pressão portal correlacionou-se com grau de fibrose hepática $(r=0,82 ; n=45)$ e seus níveis nos grupos fibrose septal $(10,8 \pm 1,2$ $\left.\mathrm{cm} \mathrm{H}_{2} \mathrm{O}\right)$ e cirrose $\left(13,6 \pm 3,1 \mathrm{~cm} \mathrm{H}_{2} \mathrm{O}\right)$ foram estatisticamente superiores aos observados no grupo-controle $\left(7,9+1,5 \mathrm{cmH}_{2} \mathrm{O}\right)$ e no grupo com fibrose perivenular $\left(9,1+0,8 \mathrm{cmH}_{2} \mathrm{O}\right)$. A concentração de laminina no sangue periférico dos cirróticos $(40,0 \pm 18,7 \mu \mathrm{g} / \mathrm{dL})$ foi significativamente superior aos grupos-controle $(13,8 \pm 12,1 \mu \mathrm{g} / \mathrm{dL})$, fibrose perivenular $(19,1 \pm 15,5)$ e fibrose septal $(22,2 \pm$ $27,0 \mu \mathrm{g} / \mathrm{dL})$. Laminina circulante correlacionou-se de maneira significativa com o grau de fibrose hepática $(\mathrm{r}=0,59 ; \mathrm{n}=49)$ e com os níveis de pressão portal $(r=0,29 ; n=45)$. Conclusões - Na intoxicação crônica experimental pelo tetracloreto de carbono, níveis circulantes de laminina correlacionam-se mais com o grau de fibrose hepática do que com os níveis de hipertensão portal.
\end{abstract}

DESCRITORES - Laminina. Tetracloreto de carbono. Matriz extracelular. Hipertensão portal. Cirrose hepática experimental. Ratos.

\section{INTRODUÇÃO}

Níveis elevados de laminina, glicoproteína da matriz extracelular (MEC), têm sido observados na circulação periférica de portadores de cirrose hepática, correlacionandose com o grau de hipertensão portal nesses pacientes ${ }^{(5,8,9)}$. Essa elevação dos níveis séricos tem sido creditada a maior deposição dessa glicoproteína no espaço de Disse, acompanhando a capilarização dos sinusóides hepáticos observada na cirrose hepática ${ }^{(6)}$. Estudos em humanos ${ }^{(7)}$ e em modelos experimentais $^{(10,11)}$ demonstram aumento na deposição dessa glicoproteína no tecido hepático, mais especificamente ao longo do espaço de Disse, à medida que progride a fibrose no fígado. Por outro lado, níveis circulantes elevados dessa glicoproteína têm sido observados em outras doenças não-cirróticas que cursam com fibrose hepática e hipertensão portal ${ }^{(17)}$.
Neste trabalho procurou-se avaliar os valores séricos de laminina na intoxicação crônica experimental pelo tetracloreto de carbono $\left(\mathrm{CCl}_{4}\right)$ e sua correlação com fibrose experimentalmente induzida, uma vez que esse modelo pode possibilitar estudos mais amplos sobre o metabolismo da laminina e os fatores que determinam sua elevação na cirrose hepática.

\section{MATERIAL E MÉTODOS}

Quarenta e nove ratos machos adultos da linhagem Wistar foram divididos em dois grupos: um grupo-controle, constituído de 16 animais submetidos a gavagem orogástrica com óleo mineral, na dose de $0,5 \mathrm{~mL}$ semanal, e um grupo tratado com $\mathrm{CCl}_{4}$ composto por 33 animais, que foram submetidos a gavagem com solução de $\mathrm{CCl}_{4}$ a $8 \%$ em óleo mineral, com dose inicial de $0,5 \mathrm{~mL}$ semanal e doses

Disciplina de Gastroenterologia - Departamento de Medicina da Universidade Federal de São Paulo - Escola Paulista de Medicina, São Paulo, SP Endereco para correspondência: Dra. Lindalva Batista Neves - Rua Otávio Nébias, 156 - ap. 141 - 04002-011 - São Paulo, SP. 
subseqüentes calculadas de acordo com o ganho ou a perda de peso, seguindo modelo previamente descrito ${ }^{(24)}$. Entre a $6^{\mathrm{a}}$ e a $16^{\mathrm{a}}$ semana de experimento, semanalmente, um rato do grupo-controle e quatro ratos da mesma idade, mas tratados com $\mathrm{CCl}_{4}$, foram sacrificados, após cateterização da veia porta com medida da pressão portal em coluna líquida contendo solução salina $0,9 \%$. Amostras de sangue foram coletadas na veia femoral, centrifugadas e armazenadas a $-20^{\circ} \mathrm{C}$, até a determinação da laminina sérica.

Fragmentos de tecido hepático foram fixados em solução de formol 10\% tamponado para análise histológica após colorações pelos métodos de hematoxilina-eosina (HE), tricrômio de Masson, reticulina, picrus sirius e prata de gomori. A fibrose hepática foi classificada de forma semi-quantitativa em cruzes, onde:

$(0)$ ausência de fibrose,$(+)=$ fibrose perivenular, $(++)=$ fibrose septal incompleta, $(+++)=$ fibrose septal completa $\mathrm{e}(++++)=$ cirrose, de acordo com classificação utilizada previamente ${ }^{(2,15)}$.

A determinação da concentração de laminina circulante foi realizada pela técnica de ensaio imunoenzimático (ELISA) de duplo anticorpo $^{(21)}$, utilizando-se anticorpo antilaminina ( $\left.\alpha \mathrm{LNM}\right)$, desenvolvido a partir de laminina isolada de tumor de camundongo Engelbreth-Holm-Swarm (EHS) ${ }^{(30)}$. Os anticorpos foram purificados em coluna de afinidade Sepharose $\mathrm{CNBr} 4 \mathrm{~B}$ ativada, utilizados na titulação de 1/100. As determinacões séricas foram realizadas em triplicatas e a leitura da reação realizada em espectrofotômetro em comprimento de onda de $492 \mathrm{~nm}$ e os valores expressos em $\mu \mathrm{g} / \mathrm{dL}$.

O protocolo foi previamente analisado e aprovado pelo Comitê de Ética da Universidade Federal de São Paulo, Escola Paulista de Medicina. Os valores das concentrações séricas de laminina foram expressos como médias \pm desvios padrões (média \pm DP). Para análise

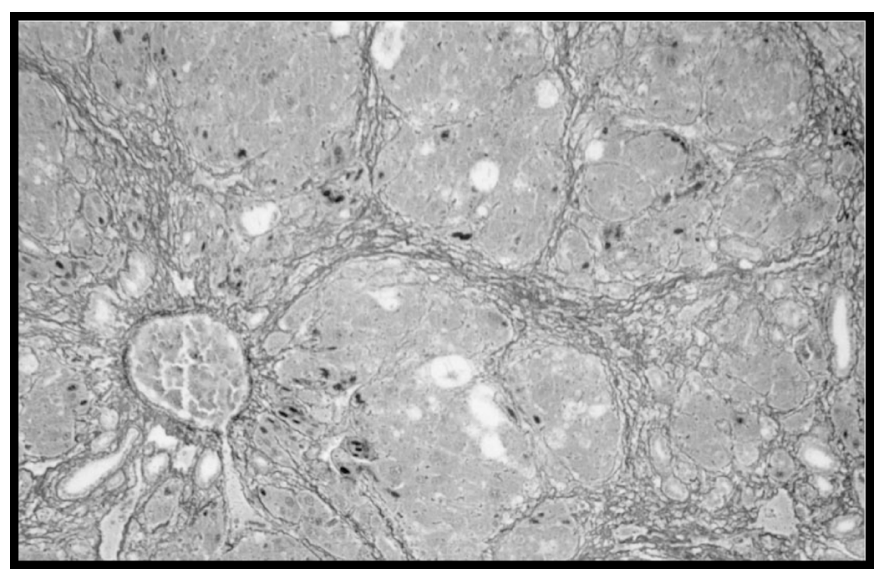

FIGURA 1 - Fotomicrografia de corte histológico de fígado de rato com intensa proliferação reticulínica, subversão da arquitetura e formação de lóbulos anômalos. Prata de Gomori (128x) comparativa dos valores de laminina nos diferentes grupos estudados, empregou-se a análise de variância não-paramétrica para postos de Kruskal-Wallis, complementada pelo teste de Dunn. A correlação entre valores de laminina e dos níveis de pressão portal com e os diferentes graus de fibrose hepática foi feita pelo índice de correlação de Spearmann, enquanto o índice de Pearson foi utilizado na correlação entre valores de laminina e de pressão portal ${ }^{(3)}$

\section{RESULTADOS}

No grupo $\mathrm{CCl}_{4}(\mathrm{n}=33)$ houve aumento progressivo do tecido fibroso hepático, iniciando-se pela fibrose perivenular, até cirrose. A cirrose foi observada (Figura 1) nos animais sacrificados a partir da 12 ${ }^{\mathrm{a}}$ semana.

Para avaliação comparativa dos valores séricos de laminina entre os grupos e pelo fato de apenas três animais terem apresentado fibrose septal incompleta, e seus valores de laminina não diferirem significativamente dos observados no grupo com fibrose septal completa, esses dois grupos foram unidos em um só, que recebeu a denominação de fibrose septal. Assim compostos os grupos, observou-se que os valores médios da concentração de laminina no sangue periférico do grupo com cirrose hepática $(40,0 \pm 18,7 \mu \mathrm{g} / \mathrm{dL})$ foi significativamente superior à média dos valores obtidos nos demais grupos: grupos-controle $(13,8 \pm 12,1 \mu \mathrm{g} / \mathrm{dL})$, fibrose perivenular $(19,1$ $\pm 15,5 \mu \mathrm{g} / \mathrm{dL})$ e fibrose septal $(22,2 \pm 27,0 \mu \mathrm{g} / \mathrm{dL})$ (Tabela 1$)$. Houve uma correlação significativa entre os níveis de laminina e o grau de fibrose hepática ( $\mathrm{rs}=0,59 ; P<0,01 ; \mathrm{n}=49 ;$ Figura 2 ). Houve também correlação significativa dos níveis de pressão portal com os diferentes graus de fibrose hepática ( $\mathrm{rs}=0,82 ; P<0,001 ; \mathrm{n}=49$; Figura 3 ) e com os níveis séricos de laminina $(\mathrm{r}=0,29 ; P<0,05 ; \mathrm{n}=45$; Figura 4$)$.

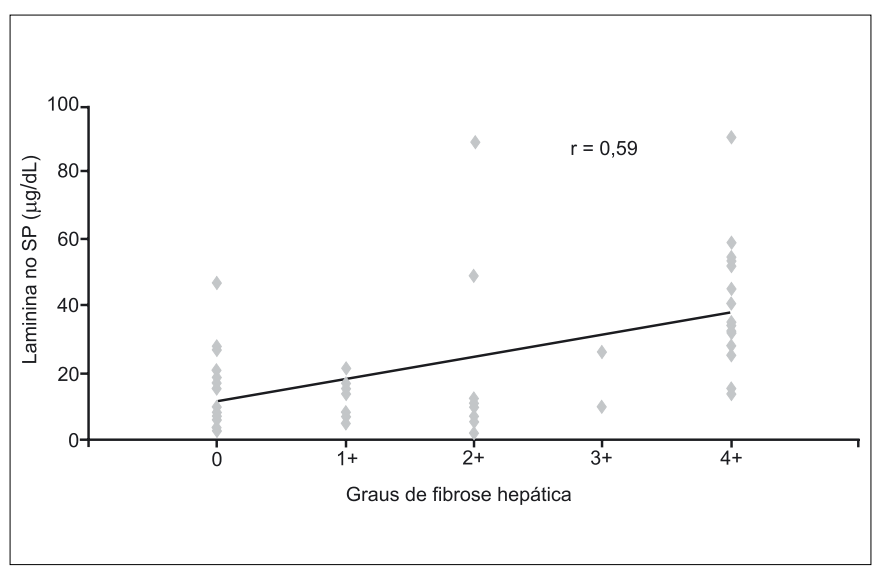

FIGURA 2 - Correlação entre concentrações de laminina no sangue periférico (SP) e graus de fibrose hepática em ratos controles e tratados com $\mathrm{CCl}_{4}(\mathrm{n}=49 ; P<0,01)$ 


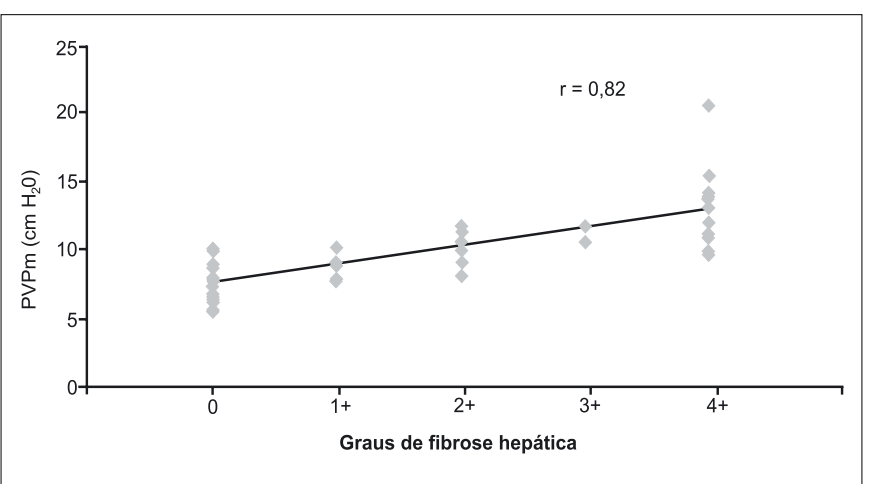

FIGURA 3 - Correlação entre pressões na veia porta e graus de fibrose hepática em ratos controles e tratados com $\mathrm{CCl}_{4}$ $(\mathrm{n}=49 ; P<0,001)$

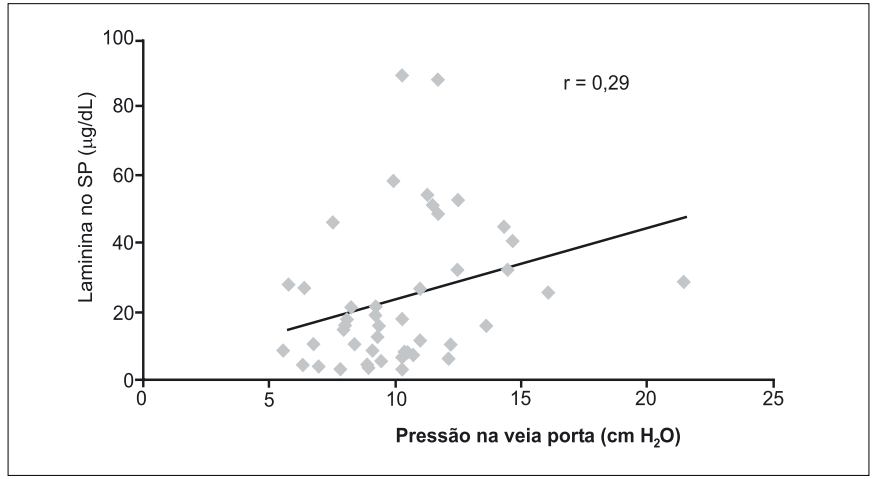

FIGURA 4 -Correlação entre concentrações de laminina sérica no sangue periférico $(\mathrm{SP})$ e pressões na veia porta $(\mathrm{cm}$ $\left.\mathrm{H}_{2} \mathrm{O}\right)$ em ratos controles e tratados com $\mathrm{CCl}_{4}(\mathrm{n}=45$ $P<0,05)$

TABELA 1 - Médias e desvios-padrões das concentrações séricas de laminina no sangue periférico de ratos controles e tratados com tetracloreto de carbono, de acordo com os graus de fibrose hepática

\begin{tabular}{lccc}
\hline Controle $(\mathbf{n}=\mathbf{1 6})$ & Fibroseperivenular $(\mathbf{n}=\mathbf{7})$ & Fibroseseptal $(\mathbf{n}=\mathbf{1 0})$ & Cirrose $(\mathbf{n}=\mathbf{1 6})$ \\
\hline $13,8 \pm 12,1 \mu \mathrm{g} / \mathrm{dL}$ & $19,1 \pm 15,5 \mu \mathrm{g} / \mathrm{dL}$ & $22,2 \pm 27,0 \mu \mathrm{g} / \mathrm{dL}$ & $40,0 \pm 18,7 \mu \mathrm{g} / \mathrm{dL}$ \\
\hline
\end{tabular}

\section{DISCUSSÃO}

O modelo experimental utilizado reproduz a fibrose hepática que ocorre em pacientes cirróticos e apresenta alterações histológicas, imunohistoquímicas e ultra-estruturais da matriz extracelular bem caracterizadas $^{(11,12,16,18,27)}$. O grande problema durante a indução da fibrose e cirrose pelo $\mathrm{CCl}_{4}$ nesses animais é a variação de resposta à toxina, em função da idade e sexo do animal, além da via, tempo de administração e dose da substância tóxica ${ }^{(18,19,20)}$. Por isto, além de padronizar as condições do experimento, optou-se por agrupar os animais estudados em função da fibrose hepática observada à histologia e não pelo tempo de intoxicação ou número de injeções de $\mathrm{CCl}_{4}$.

$\mathrm{O} \mathrm{CCl}_{4}$ leva a repetidos fenômenos necroinflamatórios no fígado, que evoluem com alterações fibróticas, inicialmente focais e posteriormente difusas, resultando em aumento da deposição de elementos do tecido conjuntivo e da pressão na veia porta ${ }^{(20,29,31)}$.

A análise histológica do tecido hepático mostrou as alterações características do modelo que validaram a classificação de fibrose utilizada $^{(24,16,19,28)}$.

A despeito da ampla utilização dos valores séricos de laminina nas doenças hepáticas e da correlação entre níveis séricos e pressão $\operatorname{portal}^{(5,8,9)}$, resta saber a exata participação do fígado na elevação de suas concentrações e a relação com a doença hepática. Diante da grande distribuição de laminina nas membranas basais do organismo e a pequena participação na MEC do fígado, algumas questões necessitam ser investigadas e esclarecidas. A correlação entre pressão portal e níveis de laminina sérica na doença hepática crônica não tem sido observada por todos os autores ${ }^{(1)}$.
No presente estudo utilizou-se anticorpo policlonal antilaminina de camundongo ( $\alpha \mathrm{LNM})$ que apresenta extensa reação cruzada com a laminina de rato ${ }^{(14,23,26)}$. A média das concentrações de laminina sérica nos ratos-controle foi próximo ao observado na literatura ${ }^{(14)}$. Esses valores aumentam em média, até quatro vezes em animais com cirrose hepática. Embora haja tendência a aumento progressivo da laminina, com o grau de fibrose, apenas animais cirróticos apresentam valores estatisticamente superiores aos controles. Houve correlação estatisticamente significativa entre concentração de laminina no sangue periférico e nível de pressão portal, porém com baixo grau de inter-relação entre si $\left(r^{2}=0,09\right)$, pela grande dispersão de valores tanto no grupo-controle, como no grupo $\mathrm{CCl}_{4}$. Por outro lado, a correlação entre a laminina no sangue periférico e graus de fibrose hepática, mostra melhor coeficiente de correlação $(\mathrm{rs}=0,59)$ do que com a pressão portal. Esses achados associados a excelente correlação entre hipertensão portal e grau de fibrose $(\mathrm{rs}=0,82)$ sugerem que níveis de laminina sérica circulante e de pressão portal não tenham relação causa-efeito e os valores de laminina sejam apenas reflexo do grau de fibrose hepática, fator este sim, determinante ou mais diretamente relacionado com hipertensão portal.

Torna-se óbvia a necessidade de avaliação do grau de deposição de laminina no fígado desses animais tanto nos sinusóides, quanto nos septos fibrosos para avaliar se nesses animais há correlação entre a capilarização dos sinusóides e a deposição de laminina no espaço de Disse e se o "turnover" alterado dessa glicoproteína seria responsável pela correlação entre seus valores séricos e a pressão portal, como observado em portadores de fibrose e cirrose hepáticas $^{(4,5,13,22,25)}$. 
Neves LB, Catarino RM, Silva MRR, Parise ER. Increased serum levels of laminin in the experimental cirrhosis induced by carbon tetrachloride. Arq Gastroenterol 2003;40(3):173-176.

ABSTRACT - Background - Serum laminin has been correlated with portal hypertension and sinusoid capillarization in chronic liver diseases. Little is known about its dynamics in liver diseases. Aim - To investigate the levels of serum laminin in experimental cirrhosis induced by carbon tetrachloride, as well as to correlate its level with the degree of hepatic fibrosis and portal hypertension. Material and Methods - Forty-nine albino Wistar rats were studied. Twenty-three were treated with carbon tetrachloride solution at $8 \%$ and 16 were kept as controls. Between the $6^{\text {th }}$ and $16^{\text {th }}$ weeks, all animals were sacrificed, submitted to measurement of portal pressure and blood sampling of the femoral veins. Liver fragments were fixed for light microscopic studies. Hepatic fibrosis was classified as perivenular fibrosis, complete and incomplete septal fibrosis and cirrhosis. Determination of laminin concentration was performed by ELISA with an antibody against laminin isolated from Engelbreth-Holm-Swarm tumor. Results - The portal pressure was correlated with the degree of hepatic fibrosis $(\mathrm{rs}=0.82 ; \mathrm{n}=45)$. Its levels in septal fibrosis $\left(10.8 \pm 1.2 \mathrm{~cm} \mathrm{H}_{2} 0\right)$ and cirrhosis $\left(13.6 \pm 3.1 \mathrm{~cm} \mathrm{H}_{2} 0\right)$ were statistically higher when compared to control $(7.9 \pm 1.5 \mathrm{~cm} \mathrm{H} 20)$ and perivenular fibrosis $(9.1 \pm 0.8 \mathrm{~cm} \mathrm{H}$ ) groups. Peripheral blood laminin concentration in cirrhosis (40.0 $\pm 18.7 \mu \mathrm{g} / \mathrm{dL})$ was significantly higher when compared to control $(13.8 \pm 12.1 \mu \mathrm{g} / \mathrm{dL})$, perivenular fibrosis $(19.1 \pm 15.5 \mu \mathrm{g} / \mathrm{dL})$ and septal fibrosis $(22.2$ $\pm 27.0 \mu \mathrm{g} / \mathrm{dL})$ groups. The circulating laminin was correlated to the degree of hepatic fibrosis $(\mathrm{rs}=0.59 ; \mathrm{n}=49)$ and to portal pressure $(\mathrm{r}=0.29 ; \mathrm{n}=45)$ Conclusions - In the chronic carbon tetrachloride intoxication, laminin levels are better correlated with the development of hepatic fibrosis than with portal hypertension.

HEADINGS - Laminin. Carbon tetrachloride. Extracellular matrix. Hypertension, portal. Liver cirrhosis, experimental. Rats.

\section{REFERÊNCIAS BIBLIOGRÁFICAS}

1. Bahr MJ, Böker KH, Horn W, Gunzler V, Manns MP. Serum laminin P1 levels do not reflect critically elevated portal pressure in patients with liver cirrhosis. Hepatogastroenterology 1997;44:1200-5.

2. Chehter L. Redução da fibrose hepática induzida pelo tetracloreto de carbono, pela administração prévia de vitamina A [dissertação]. São Paulo: Escola Paulista de Medicina; 1987

3. Glantz SA. Primer of biostatistics. $4^{\text {th }}$ ed. New York: Mac Graw-Hill; 1997. $473 \mathrm{p}$.

4. Gressner AM, Tittor W, Negwer A, Pick-Kober KH. Serum concentration of laminin and aminoterminal propeptide of type III procollagen and in relation to the portal venous pressure of fibrotic liver diseases. Clin Chim Acta 1986;161:249-58

5. Gressner AM, Tittor W. Serum laminin. Its concentration increases with portal hypertension in cirrhotic liver disease. Klin Wochensch 1986;64:1240-8.

6. Gressner AM, Tittor W, Kropf J. The predictive value of serum laminin for portal hypertension in chronic liver disease. Hepatogastroenterology 1988;35:95-100.

7. Hahn EG, Wich G, Pencev D, Timpl R. Distribution of basement membrane proteins in normal and fibrotic human liver: collagen type IV, laminin and fibronectin. Gut 1980;21:63-71

8. Kondo M, Miszputen SJ, Leite-Mor MM, Parise ER. The predictive value of serum laminin for the risk of variceal bleeding related to portal pressure levels. Hepatogastroenterology 1995;42:542-5.

9. Mal F, Hartmann DJ, Trinchet JC, Lacombe F, Ville G, Beaugrand M. Laminine sérique et pression portale au cours de la cirrhose alcoolique. Une etude chez 39 malades. Gastroenterol Clin Biol 1988;12:841-4.

10. Martinez-Hernandez A. The hepatic extracellular matrix: I. Electron immunohistochemical studies in normal rat liver. Lab Invest 1984;51:57-74.

11. Martinez-Hernandez A. The hepatic extracellular matrix: II. Electron immunohistochemical studies in rats with CCl4-induced cirrhosis. Lab Invest 1985;53:166-86.

12. Martinez-Hernandez A, Martinez J. The role of capillarization in hepatic failure: studies in carbon tetrachloride-induced cirrhosis. Hepatology 1991;14:864-74.

13. Martinez-Hernandez A, Martinez-Delgado F, Amenta PS. The extracellular matrix in hepatic regeneration: localization of collagen types I. III, IV, laminin and fibronectin. Lab Invest 1991;64:157-66.

14. Monteiro GS. Desenvolvimento de ensaio para determinação sérica de laminina e avaliação do seu valor prognóstico em cânceres humanos [dissertação]. São Paulo: Escola Paulista de Medicina; 1990.

15. Nouchi T, Worner TM, Sato S, Lieber CS. Serum procollagen type III N-terminal peptides and laminin P1 peptide in alcoholic liver disease. Alcohol Clin Exp Res 1987;11:287-91.
16. Orrego H, Blendis LM, Crossley IR, Medline A, MacDonald A, Ritchie S, Israe Y. Correlation of intrahepatic pressure with collagen in the Disse space and hepatomegaly in humans and in the rat. Gastroenterology 1981;80:546-56.

17. Parise ER, Rosa H. Serum laminina in hepatic schistosomiasis. Trans R Soc Trop Med Hyg 1992;86:179-81.

18. Pérez-Tamayo R. Is cirrhosis of the liver experimentally produced by $\mathrm{CCl} 4 \mathrm{na}$ adequate model of human cirrhosis? Hepatology 1983;3:112-20.

19. Proctor E, Chatamra K. Controlled induction of cirrhosis in the rat. Br J Exp Path 1983;64:320-30

20. Recknagel RO, Ghoshal AK. Quantitative estimation of peroxidative degeneration of rat liver microsomal and mitochondrial lipids after carbon tetrachloride poisoning. Exp Mol Pathol 1966;5:413-26.

21. Rennard IS, Berg R, Martin RG, Foidart JM, Robey PG. Enzime-linked immunoassay (ELISA) for connective tissue components. Anal Biochem 1980;104:205-14.

22. Risteli J, Rohde H, Timpl R. Sensitive radioimmunoassays for 7S collagen and laminin: applications to serum and tissue studies of basement membrane. Anal Biochem 1981;113:372-8.

23. Risteli L, Timpl R. Isolation and characterization of pepsin fragments of laminin from human placental and renal membranes. Biochem J 1981;193:749-55.

24. Rosa H, Parise ER, Paranhos FRL, Braga AL, Freitas ASA, Hidalgo AN, LeiteMor MB. Controlled production of cirrhosis in the rat liver. Arq Gastroenterol 1991;28:39-43

25. Schaffner F, Popper H. Capilarization of hepatic sinusoids in man Gastroenterology 1963;44:239-42

26. Schechter GB, Lopes JD. Two-site immunoassays for the measurement of serum laminin: correlation with breast cancer staging and presence of auto-antibodies. Braz J Med Biol Res 1990;23:141-9.

27. Seyer JM. Intersticial collagen polymorphism in rat liver with CCl4-induced cirrhosis. Biochem Biophys Acta 1980;629:490-8.

28. Shibayama Y, Nakata K. The role of septal fibrosis in development of hepatic circulatory disturbance in the presence of liver cell enlargement. Liver 1992;12:84-9

29. Suematsu M, Kato S, Ishii H, Asako H, Yanagisawa T, Suzuki H, Oshio C, Tsuchiya M. Intralobular heterogeneity of carbon tetrachloride-induced oxidative stress in perfused rat liver visualized by digital imaging fluorescence microscopy. Lab Invest 1991;64:167-73

30. Timpl R, Rohde G, Robey PG, Rennart SI, Foidart JM, Martin GR. Laminin glycoprotein from basement membranes. J Biol Chem 1979;254:9933-37.

31. Tsukamoto H, Matsuoka M, French SW. Experimental models of hepatic fibrosis a review. Semin Liver Dis 1990;10:56-63. 